Utah State University

DigitalCommons@USU

Reports

Utah Water Research Laboratory

January 1995

\title{
Locally Weighted Polynomial Regression: Parameter Choice and Application to Forecasts of the Great Salt Lake
}

Upmanu Lall

Young-II Moon

Ken Bosworth

Follow this and additional works at: https://digitalcommons.usu.edu/water_rep

Part of the Civil and Environmental Engineering Commons, and the Water Resource Management

Commons

\section{Recommended Citation}

Lall, Upmanu; Moon, Young-II; and Bosworth, Ken, "Locally Weighted Polynomial Regression: Parameter Choice and Application to Forecasts of the Great Salt Lake" (1995). Reports. Paper 243.

https://digitalcommons.usu.edu/water_rep/243

This Report is brought to you for free and open access by the Utah Water Research Laboratory at DigitalCommons@USU. It has been accepted for inclusion in Reports by an authorized administrator of DigitalCommons@USU. For more information, please contact digitalcommons@usu.edu.

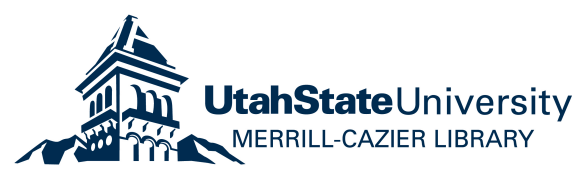




\title{
LOCALLY WEIGHTED POLYNOMIAL REGRESSION:
}

\section{PARAMETER CHOICE AND APPLICATION TO FORECASTS \\ OF THE GREAT SALT LAKE}

\author{
By: \\ Upmanu Lall, Young-II Moon and Ken Bosworth \\ Working Paper WP-95-HWR-UL/014 \\ May 1995
}

Utah Water Research Laboratory

Utah State University

Logan, UT 84322-8200

(801) $797-3155$

Fax (801) 797-3663 


\title{
Locally Weighted Polynomial Regression: Parameter Choice and Application to Forecasts of the Great Salt Lake
}

\author{
by \\ Upmanu Lall and Young-II Moon \\ Utah Water Research Lab. and Dept. of Civil and Environ. Eng. \\ Utah State University, Logan UT 84322-8200 \\ and \\ Ken Bosworth \\ Department of Mathematics, Idaho State University, Pocatello ID 83209-8085
}

\begin{abstract}
Relationships between hydrologic variables are often nonlinear. Usually the functional form of such a relationship is not known a priori. A multivariate, nonparametric regression methodology is provided here for approximating the underlying regression function using locally weighted polynomials. Locally weighted polynomials consider the approximation of the target function through a Taylor series expansion of the function in the neighborhood of the point of estimate. Cross validatory procedures for the selection of the size of the neighborhood over which this approximation should take place, and for the order of the local polynomial to use are provided and shown for some simple situations. The utility of this nonparametric regression approach is demonstrated through an application to nonparametric short term forecasts of the biweekly Great Salt Lake volume. Blind forecasts up to four years in the future using the 1847-1993 time series of the Great Salt Lake are presented.
\end{abstract}




\section{Introduction}

Most hydrologists are familiar with linear regression and its use for developing a relationship between two or more variables. The general linear model (where the variables are presumed to be linearly related after applying some predetermined transform) is used as a building block in many activities ranging from spatial surface estimation, missing value imputation, sediment load estimation to autoregressive time series modeling. Often, such a procedure is not very satisfying. The choice of an appropriate transform to use may not be obvious, and scatterplots of the data may not visually support the assumed model. An alternative to such regression approaches that is capable of representing relatively complex relationships between variables, through "local" or pointwise approximation of the underlying function, is presented here. Procedures that determine model parameters automatically from the data, and also allow the user to choose between a parametric (i.e. linear model) and a nonparametric model (i.e., a local linear model) are provided.

There has been a surge in the development and application of nonparametric regression and density estimation methods in the last decade as computational resources have become more accessible. Some monographs that make this literature accessible are by Silverman (1986), Eubank (1988), Härdle (1989). Examples of nonparametric estimators include orthogonal series expansions, moving averages, splines, kernel, and nearest neighbor estimators. The reader is referred to Lall (1995) for a recent review of the application of nonparametric methods in hydrology. Some attributes of these methods are :

(1) The estimator can often be expressed as a weighted moving average of the observations.

(2) The estimates are defined locally or using data from a small neighborhood of each point of estimate.

(3) Consequently, they can approximate a wide class of target, underlying functions.

(4) The nonparametric estimator has parameters that control the local weights and the size of the neighborhood used for estimation. However, unlike linear or parametric regression, where the parameters (e.g., intercept and slope) are sufficient to provide an estimate at any point, the nonparametric estimator needs the observations in the neighborhood to provide an estimate at any 
point. For example the parameter of a moving average is the number of points (e.g., 3) to average over. One still needs the three points surrounding the point of estimate to report the answer. By contrast, once a linear regression has been evaluated, the parameters are all one needs to provide an estimate at any point. In the parametric case, the overall behavior is of an assumed form (e.g., linear or log-linear), and the parameters of this global form are all one needs to estimate. The parameters of a nonparametric model thus have a different role, since no global form is assumed, and the parameters merely control how local averages are to be formed.

Background information on multivariate, locally weighted polynomial regression is provided in the next section. Methods for parameter selection and the estimation of error variances and confidence intervals are presented next. An algorithm for local regression is then summarized. An application of local regression to time series forecasting is presented. A biweekly record of the Great Salt Lake volume from 1847-1993 was used for the forecasts. The dynamics of the time series of the Great Salt Lake has been studied by Sangoyomi (1993), Lall et al (1995a,b) and Abarbanel and Lall (1995). This work suggests that the dynamics of the Great Salt Lake (GSL) is nonlinear, and possibly chaotic, and demonstrated the success of a nonparametric regression scheme (Multivariate Adaptive Regression Splines, or MARS, due to Friedman (1991)) for forecasting the GSL for up to 4 years ahead during extreme hydrologic periods. The local regression scheme presented here is a computationally faster alternative to MARS, and is easier to explain and analyze.

\section{Background}

Consider a general regression model given as :

$$
\mathrm{y}_{\mathrm{i}}=\mathrm{f}\left(\mathbf{x}_{\mathrm{i}}\right)+\mathrm{e}_{\mathrm{i}} \quad \mathrm{i}=1, \ldots, \mathrm{n}
$$

where, $\mathbf{x}$ is a $M$-vector of $M$ explanatory variables, $y$ is the "response" variable, $f($.$) represents the$ underlying functional relationship between $y$ and $\mathbf{x}, \mathrm{e}_{\mathbf{i}}$ are noise or measurement errors, that may or may not depend on $x_{i}$, and $n$ is the number of observations.

Linear regression considers the case where the function $f($.$) is linear in x$, i.e., $f(x)=x \beta$, where $\beta$ is a $\mathrm{M}$-vector of coefficients that do not depend on $\mathbf{x}$. Theoretical methods for parameter selection, analysis of variance, hypothesis testing, estimating confidence and prediction intervals, 
outlier identification and other related statistical activities are well developed and understood for linear regression (Stuart and Ord (1991)). Statistical software is readily available to perform these computations. Consequently, these methods see widespread use. Nonlinear relationships between $\mathbf{x}$ and $\mathbf{y}$ are admitted in this framework through prior transforms applied to $\mathbf{x}$, and/or $\mathbf{y}$. For instance, the components of $x$ may include polynomial terms $\left(1, v, v^{2} \ldots\right)$ in an original variable $v$. The Box-Cox family of power transformations is often used in hydrology (see Helsel and Hirsch, 1992) to select an appropriate transform. There are a number of difficulties with such an approach. These include (1) the inability to find an appropriate transform for a given data set, (2) bias in the estimates upon backtransforming, (3) non-unique selection of applicable transforms for a given data set, which can lead to an unquantifiable uncertainty of estimate (particularly while extrapolating the data, which is the usual application!), and (4) reconciliation of the distribution of errors in the transformed and original coordinates relative to the underlying error distribution.

An approach for the pointwise estimation of the unknown function $f($.$) from data, based on$ a local Taylor series expansion of $f(x)$ at the point of estimate $\mathbf{x}$, was proposed by Macauley (1931). Cleveland (1979), Cleveland and Devlin (1988) and Cleveland et al (1988) pioneered this idea into a statistical methodology for local approximation of functions from data. Recently (e.g., Hastie and Loader (1993), Fan and Gijbels (1992), Müller (1987), Lejeune (1985)), these methods have been recognized as very useful generalizations of kernel regression (weighted moving averages). Applications to a suite of statistical estimation problems are emerging. Our presentation here is specific to the estimator we describe. The material presented here builds directly on the method of Cleveland and Devlin (1988), and the developments in Lall et al (1995). The reader is referred to a recent monograph by Wand and Jones (1995) for general background on the methods.

Generally, the strategy is to choose a certain number, $\mathrm{k}$, of nearest neighbors (in terms of Euclidean distance) of the estimation point $\mathbf{x}$, and to form the estimate $\hat{\mathrm{f}}(\mathbf{x})$ through a locally weighted, polynomial regression over the $(x, y)$ data that lie in the neighborhood. Consider the general regression model described in (1). The sampling locations $\mathbf{x}_{\mathbf{i}}$ are usually not regularly spaced. We assume the $e_{i}$ are uncorrelated, mean zero, random variables, assumed to be approximately identically distributed in the $\mathrm{k}$ nearest neighborhood of the point of estimate.

To motivate the local polynomial regression technique for approximating a wide class of functions $f($.$) , first suppose that the errors e_{i}$ are identically zero. Then locally, about some $x^{*}$, we 
can estimate $f($.$) using a (multivariate) polynomial in \mathbf{x}$ which is chosen to interpolate $\mathrm{y}_{\mathrm{i}}=\mathrm{f}\left(\mathbf{x}_{\mathrm{i}}^{*}\right)$ at $\mathbf{x}_{i}^{*}, i=1 . . k$, in the $k$ nearest neighborhood of $\mathbf{x}^{*}$. The data indices $i=1 \ldots k$ are arranged so that the data $\mathbf{x}_{1}^{*}$ are arranged in order of increasing distance from $x^{*}$. Here, we assume the underlying $f$ is locally a smooth (i.e. continuous and differentiable to some order) function. In the univariate setting we can write the following Newton-Taylor expansion

$$
\begin{aligned}
f(x)= & f\left[x_{1}^{*}\right]+f\left[x_{1}^{*}, x_{2}^{*}\right]\left(x-x_{1}^{*}\right)+\ldots+f\left[x_{1}^{*}, x_{2}^{*}, \ldots, x_{k}^{*}\right]\left(x-x_{1}^{*}\right)\left(x-x_{2}^{*}\right) \ldots\left(x-x_{k-1}^{*}\right) \\
& +f^{(k)}(\zeta)\left(x-x_{1}^{*}\right) \ldots\left(x-x_{k-1}^{*}\right)\left(x-x_{k}^{*}\right) / k ! \\
= & p_{k}(x)+f^{(k)}(\zeta)\left(x-x_{1}^{*}\right) \ldots . .\left(x-x_{k}^{*}\right) / k !
\end{aligned}
$$

where the points $x$ and $\zeta$ are in the convex hull of $\left\{x_{1}^{*}, \ldots, x_{k}^{*}\right\}$, and $f\left[x_{1}^{*}, x_{2}^{*}, \ldots, x_{j}^{*}\right]$ is the $(j-1)^{\text {th }}$ divided difference (defined as the coefficient of the term $x^{j-1}$ of the polynomial of degree $j-1$ that agrees with $\left.f\left(x_{1}\right), f\left(x_{2}\right) \ldots f\left(x_{j}\right)\right)$ of $f($.$\left.) at the sample points \left\{x_{1}^{*}, x_{2}^{*}, \ldots, x_{j}^{*}\right\}\right)$. The point $\zeta$ is generally unknown, but guaranteed to exist by the mean value theorem provided $f($.$) is \mathrm{C}^{\mathrm{k}}$ smooth (i.e., continuous and differentiable to order $\mathrm{k}$ ). We notice that if the sample points are closely grouped (i.e., $\left|\mathrm{x}-\mathrm{x}_{\mathrm{k}}^{*}\right|$ is small) and $\mathrm{f}\left(\right.$.) is locally $\mathrm{C}^{\mathrm{k}}$ smooth (i.e., the $\mathrm{k}^{\text {th }}$ derivative is finite), then the last term in the above equality can be neglected, and one obtains the local $\mathrm{k}^{\text {th }}$ order polynomial approximation $\mathrm{p}_{\mathrm{k}}(\mathrm{x})$ to $\mathrm{f}(\mathrm{x})$.

The choice of the $\mathbf{x}_{1}^{*}$ 's for any given $\mathbf{x}$ can be problematic in several ways. First, $\mathbf{x}^{*}$ should be "centered" within the set $\left\{\mathbf{x}_{1}^{*}, \mathbf{x}_{2}^{*}, \ldots, \mathbf{x}_{\mathrm{k}}^{*}\right\}$. This is not a problem if the sample points $\mathbf{x}_{1}^{*}$ are uniformly distributed, and $\mathbf{x}$ is not near the boundary of the region. However, in situations where one has non-uniform sampling, and when one estimates $f($.$) near the boundary, we expect a$ deterioration in the estimate of $f($.$) provided by \mathrm{p}_{\mathrm{k}}($.$) . Secondly, there is a choice of how many$ points, $\mathrm{k}$, to use in building the estimator $\mathrm{p}_{\mathrm{k}}($.$) . Choosing too few neighbors about \mathrm{x}$ results in loss of information contained in higher derivatives; choosing too many neighbors (resulting in a higher degree polynomial) yields an estimator with too many degrees of freedom, allowing too much variation between the interpolation points. That is, the true, but unknown local behavior of $f$ near $x^{*}$ may not be well represented by bringing in interpolation points "far away" from $x^{*}$. Thus, even working with error free, perfect data, the idea of representing $f$ by a local polynomial requires a means of balancing the trade-off occurring between choosing small local neighborhoods 
(i.e., small $\mathrm{k}$ ), resulting in a possibly oversmoothed or biased estimate, and choosing large neighborhoods, resulting in superfluous degrees of freedom. Asymptotically, (i.e., as the sample size $n \rightarrow \infty$, one would decrease $k$, since the distance $\left|x-x_{k}^{*}\right|$ will approach zero.

With the introduction of noise, the above procedure can be readily adapted by requiring that an estimate of $f($.$) near a given x^{*}$ be obtained by performing a local least squares polynomial regression on the $\mathrm{k}$ nearest neighborhood, and using this regression polynomial's value at $\mathrm{x}^{*}$ to estimate $f\left(x^{*}\right)$. It is desirable to prescale each data vector or coordinate in $\mathbf{x}$ to have similar scale, prior to computing nearest neighbor distances. In our implementation, we consider scaling each component to have a mean 0 , and variance 1 , or to lie between 0 and 1 . Practically, linear, quadratic and cubic polynomials are useful. Using a polynomial of degree $p$, we may have $d$ parameters that need to be estimated locally. Note that $d<k$, else we can interpolate, with potentially disastrous results. In practice, we enforce $k>2 d$. It is desirable to form estimates that are "local", since the "end points" in a linear regression can have a large influence on the resulting estimate. Given these observations, and the observation that the point of estimate should be approximately centered in the locale of estimation, it is desirable to weight the local polynomial fit, such that observations close to the point of estimate are accorded a higher importance in the least squares fit, than those that lie further away in the neighborhood.

Then, the locally weighted polynomial regression at each point of estimate $x_{1}^{*}, 1=1$.np, given a $\left(n^{*} M\right)$ data matrix $\mathbf{X}$ and a $\left(n^{*} 1\right)$ response vector $\mathbf{y}$, is obtained through the solution of the weighted least squares problem:

$\operatorname{Min}\left(\mathbf{y}_{1}-\mathbf{z}_{1} \beta_{1}\right)^{\mathrm{T}} \mathrm{w}_{1}\left(\mathrm{y}_{1}-\mathrm{z}_{1} \beta_{1}\right)$

$\beta_{1}$ 
where the subscript 1 recognizes that the associated element is connected with the point of estimate $x_{1}^{*} ; \beta_{1}$ are estimates of the coefficients of the terms in the basis defined by $\mathbf{Z}_{1} ; \mathbf{z}_{1}$ is a matrix formed by augmenting $\mathbf{X}$, with columns that represent the polynomial expansion of $\mathbf{X}$ to degree $\mathrm{p}$ (including cross product terms if desired); $\mathbf{W}_{1}$ is a $\mathrm{k}^{*} \mathrm{k}$ diagonal weight matrix with elements $\mathrm{w}_{\mathrm{i}, \mathrm{l}}=\mathrm{K}\left(\mathrm{u}_{\mathrm{i}, 1}\right) / \sum_{\mathrm{j}=1}^{\mathrm{k}} \mathrm{K}\left(\mathrm{u}_{\mathrm{j}, 1}\right)$, where $\mathrm{u}_{\mathrm{i}, 1}=\mathrm{d}_{\mathrm{i}, \mathrm{l}} / \mathrm{d}_{\mathrm{k}, 1} ; \mathrm{d}_{\mathrm{i}, 1}$ is the distance from $\mathbf{x}_{1} *$ to $\mathbf{x}_{\mathrm{i}}$ using an appropriate metric, and $\mathrm{K}($.$) is a weight function.$

We have implemented a bisquare kernel $\left(K(u)=15 / 16\left(1-u^{2}\right)^{2}\right)$. The latter is recommended by Scott (1992) because of its smoothness properties. The matrices $\mathbf{y}_{1}$ and $\mathbf{Z}_{1}$ are defined over the $\mathrm{k}$ nearest neighborhood of $\mathbf{x}_{1}$ *. Singular Value Decomposition (SVD) using algorithms from Press (1989) is used to solve the linear estimation problem resulting from (3).

The reader may note that we are in the familiar territory of linear regression, and will hence have the usual statistical tools available to us. The coefficients $\beta_{1}$ are obtained as:

$$
\boldsymbol{\beta}_{1}=\left(\mathbf{Z}_{1}^{\mathrm{T}} \mathbf{W}_{1} \mathbf{Z}_{1}\right)^{-1} \mathbf{Z}_{1}^{\mathrm{T}} \mathbf{W}_{1} \mathbf{y}_{1}
$$

The resulting estimate of $\hat{f}\left(\mathbf{x}_{1}\right)$ is then:

$$
\hat{\mathrm{f}}\left(\mathbf{x}_{1}\right)=\mathrm{z}_{1} \beta_{1}
$$

where $z_{1}$ is the $d^{\prime} * 1$ vector formed by augmenting $x_{1}$ with polynomial terms to order $p$, and retaining the terms for which $\beta_{\mathrm{j}}$ are found to be significantly different from 0 .

Expressions for the asymptotic bias and variance of an estimate equivalent to (5) are presented by Wand and Jones (1995, p. 140), and are not reproduced here. Instead, we shall develop some data driven estimates of variance and mean square error of estimate, under the assumption that the number of nearest neighbors, $\mathrm{k}$, and the order of the local polynomial, $\mathrm{p}$ has been chosen appropriately. We shall consider $k$ and $p$ the "smoothing parameters" of the estimation scheme and consider the slopes $\beta_{1}$ to be "structural parameters".

The mean square error of estimate at $x_{1}$ is estimated from the local regression as:

$$
\operatorname{MSE}\left(\hat{\mathrm{f}}\left(\mathbf{x}_{1}\right)\right)=\mathbf{e}_{1}^{\mathrm{T}} \mathbf{W}_{1} \mathbf{e}_{1}
$$

Note that this is a weighted average of the squared errors from the local regression, with the same weights as those used for estimating the regression. Essentially, the mean square error of fit is localized to the point of estimate. 
An estimate of the variance of the local errors $\mathrm{e}_{\mathrm{i}, \mathrm{l}}, \mathrm{i}=1 \ldots \mathrm{k}$, is available as:

$s_{\mathrm{e}_{1}}^{2}=\mathbf{e}_{1}^{\mathrm{T}} \mathbf{W}_{1} \mathbf{e}_{1} /((\mathrm{k}-\mathrm{d}) / \mathrm{k})$

where $d$ is the number of parameters fitted.

One can thus allow an error structure that is localized to the point of estimate as well. Hypothesis tests can then be designed to see if the error variance at two points is comparable or not. Such tests can either be implemented nonparametrically (e.g., through a bootstrap (Efron and Tibishirani (1993) of the data), or parametrically with assumptions as to the probability distribution of errors.

Under the assumption that the local errors $e_{i, 1}$ are approximately normally distributed, one can construct a t-test for a hypothesis that individual coefficients $\beta_{j}$ are significantly different from zero, at a desired significance level $\alpha$. A judicious application of this $t$ test with a significance level $\alpha$, allows one to screen spurious coefficients and improve the degrees of freedom of the local fit, retaining $\mathrm{d}^{\prime}$ of the $\mathrm{d}$ original terms in the regression. The t-statistic of interest (Stuart and Ord (1991, p. 1042), with (k-d) degrees of freedom is:

$t_{j}=\beta_{j} /\left(s_{e_{1}}^{2} a_{j j}\right)^{0.5}$

where $a_{j j}$ is the $(j, j)$ element of the matrix $\left(\mathbf{Z}_{1}^{\mathrm{T}} \mathbf{W}_{1} \mathbf{Z}_{1}\right)^{-1}$.

Approximate confidence and prediction intervals for the estimate in (5) can also be obtained as for the regular linear regression model. We shall defer the presentation of these estimates until the end of the next section which focuses on the selection of the number of nearest neighbors and the order of the polynomial.

\section{Smoothing Parameter Selection and Prediction Limits}

Some of the issues that are important for picking the order of the local polynomial and for selecting the number of nearest neighbors to use were touched on in the last section. Here, we shall present the selection process we have adopted for smoothing parameter selection. It is helpful to begin with a simple univariate example. Consider the estimation of the function $f(x)=\operatorname{Sin}(x) e^{-}$ $0.2 x$, from the data $\left(x_{i}, y_{j}\right)$, generated such that the $x_{i}$ are equally spaced values from 0 to 10 , and the $y_{i}$ are then generated as $f\left(x_{i}\right)+e_{i}, i=1 . .100$, and $e_{i} \sim N(0,0.1)$. This data set, the true, underlying function, and 3 local regressions are shown in Figure 1. 


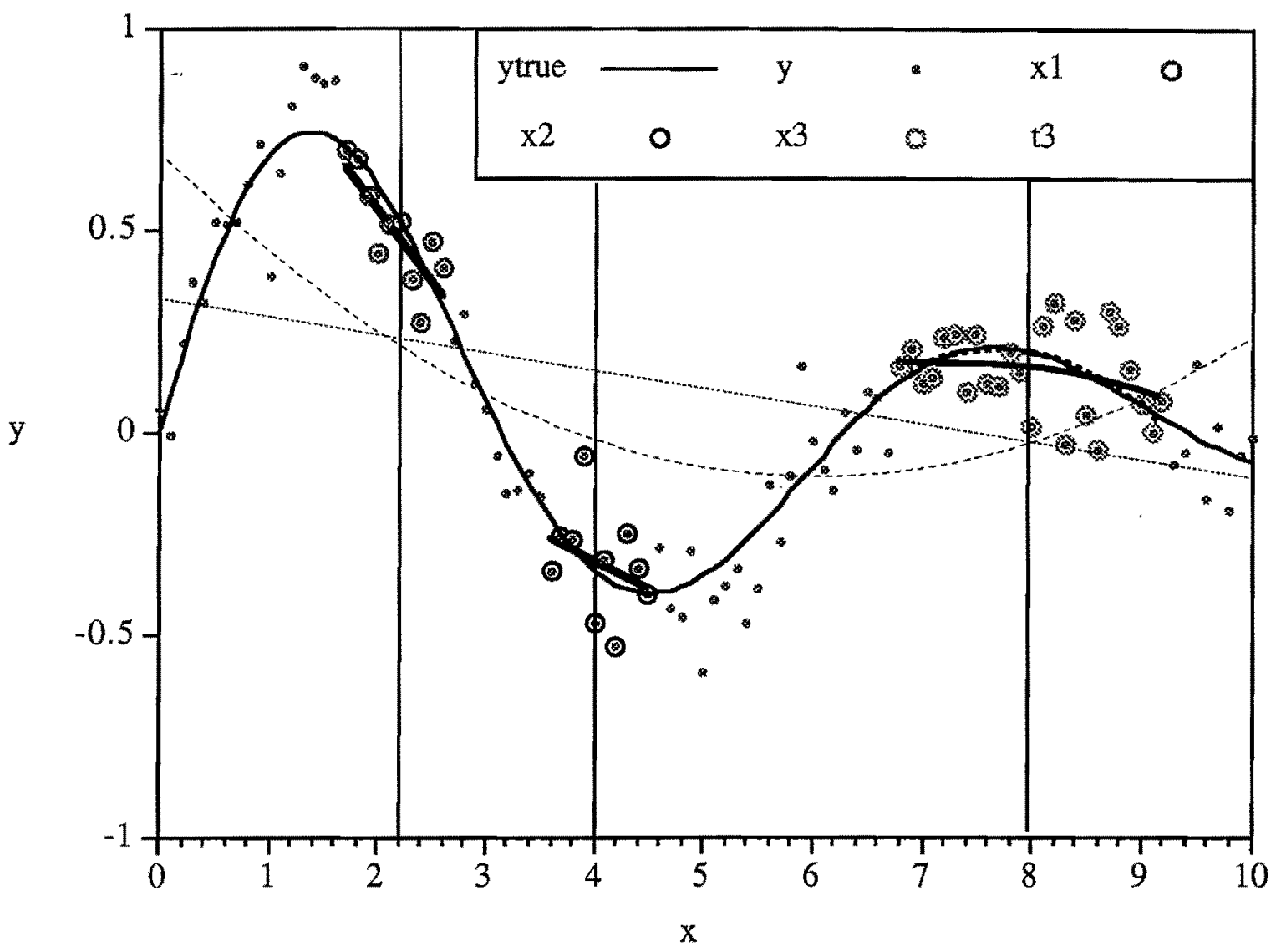

Figure 1

fllustration of local linear and local quadratic regression, with the weights $w_{i i}=1 / \mathrm{k}$. The data (small circles $)$ is 100 points generated from $y=\operatorname{Sin}(x) e^{-0.2 x}+N(0,0.1)$. The true function is shown as the solid line. The thin dotted line is a linear regression through the full data. It shows the bias incurred if a neighborhood of size 100 is used at any point. The thin dashed line is a quadratic fit through the data. It shows that the bias may be reduced by going to a higher order polynomial. Estimates are considered at 3 points, $x=2.2,4$ and 8 . Local linear fits with 10 neighbors are used at the first two points, and a locally quadratic fit with 25 neighbors is used at $x=8$. The data in each neighborhood are shown with large circles. The local fits are shown as thick solid lines. The approximation at the first two points is quite good. The estimate at $x=8$ is poorer. Since we know the true function, we can use those values with the local quadratic fit. The resulting fit at $x=8$ is shown as a thick dashed line. It is seen to coincide with the target function. Consequently, the approximation error at $x=8$ is a consequence of the local noise realization. 
We observe that the quality of the local regressions is quite good. The higher order (quadratic) fit is less biased than the linear, and the bias decreases substantially as the size of the neighborhood is reduced from 100 to 10 or 25 points. However, the problem with local regressions of increased variability of estimate due to the reduced sample size is also shown. This is exacerbated as one moves to a higher order polynomial fit with the same number of data points.

Thus, there is a trade off between bias and variance as one changes the order of the local polynomial and the number of points used to fit it. Another potential problem arises if the $x_{i}$ values are randomly sampled or are clustered in certain locations. Consider for instance the following values of $x_{i}(3 \cdot 1,3 \cdot 2,3 \cdot 5,4 \cdot 5,5,20,22,25,30,31)$. Now consider a local regression at $x=10$. In this case, the first 5 neighbors of the point of estimate are all to its left, and no information is used from the data on the right. An estimate with $k \leq 5$ will then certainly be an extrapolation of the data used. Recall from section 2, that it is desirable that the point of estimate be centered in its estimation neighborhood. Recall also that the variance of estimate of linear regression increases dramatically as one moves towards the edges of, or out of the range of data. Consequently, in the multivariate setting, we may find it desirable to devise a strategy by which we symmetrize the neighborhood of the point of estimate, and to explicitly consider the degree of extrapolation involved in a candidate local regression.

Parameter selection approaches are usually based on an estimate of the Mean Square Error of the estimation scheme. Here, we have to be careful, since one can easily choose $k$ and $p$ to drive the mean square error of fit to zero, and the associated $R^{2}$ will be 1 ! Of course, this may not say much about performance in a predictive as opposed to fitting setting. Consequently, we shall investigate some cross validatory choices of measures like mean square error for parameter selection.

A variety of estimators of Predictive Mean Square Error $P(\hat{f})$ have been proposed in the literature. Cleveland and Devlin (1988) considered Mallows $C_{p}$ in their work on local polynomial regression. $\mathrm{Li}$ (1985) discusses the theoretical foundations of this and other measures such as Ordinary and Generalized Cross Validation, the Finite Prediction Error, the AIC and the BIC. Of these the Generalized Cross Validation (GCV) statistic proposed by Craven and Wahba (1979) is of particular interest since it has performed well (Härdle $(1984,1989)$ ) in practical applications. It 
is defined as :

$\operatorname{GCV}(\hat{f})=\operatorname{MSE}(\hat{f}) /\left(n^{-1} \operatorname{tr}[\mathbf{I}-\mathbf{H}]\right)^{2}$

where

the Mean Square Error MSE $(\hat{f})=n^{-1} \sum_{i=1}^{n}\left(y_{i}-\hat{f}_{i}\right)^{2}$

$\mathbf{H}$ is the influence matrix defined through $\hat{\mathbf{f}}=\mathbf{H} \mathbf{y}$

$I$ is the identity matrix, and $t r[$.$] represents the trace of the matrix.$

Note that (11) represents a linear estimator, and the $i^{\text {th }}$ diagonal element of $\mathbf{H}$ can be thought of as the "weight" of that data point on the estimate at that point. Eubank (1988), p. 406 states that for linear regression (local or global, and on the raw variable or a polynomial in it) it is easy to show that $0 \leq h_{i i} \leq 1$. Thus if $h_{i i}$ is 1 , and the other $h_{i j}$ are 0 , we see that we have 0 degrees of freedom, and the estimate at each point is simply the original data, i.e. the model completely overfits or undersmooths. The corresponding MSE is zero, and the GCV is infinity. On the other hand if all the $h_{i j}$ are equal, the estimate at every point is the sample average of the $y_{i}$, the degrees of freedom are ( $\mathrm{n}-1$ ), since we fit one parameter, and the MSE may be large if $\mathrm{f}($.$) is not a$ constant, and for $n$ large, MSE and GCV will approach each other in magnitude. Consider also the case where the $h_{i j}$ are equal for the $\mathrm{k}$ nearest neighbors of a point and 0 elsewhere. In this case we may approximate $f($.$) better since we form a moving average of y$ values, and hence have a lower MSE. However, the degrees of freedom will only be $(\mathrm{k}-1)$, and the GCV may be larger. The denominator in (9) consequently has the role of a penalty for the effective number of parameters used in fitting the model. The effective number of parameters is determined by the number of neighbors and the number of terms in the local polynomial.

The motivation for using GCV(f) comes from a theorem proved by Craven and Wahba (1979). They showed that $\operatorname{GCV}(\hat{f})$ is a nearly unbiased estimator of $P(\hat{f})$. Eubank (1988) also shows that the GCV is closely related to the Ordinary Cross Validation (OCV) estimate of $P(\hat{\mathrm{f}})$ (p.30), and to the Akaike Information Criteria (p.40). OCV considers the Mean Square Error in dropping one observation at a time from the fitting set and then predicting it using the remaining data. It also estimates $P(\hat{f})$, but at a higher computational cost.

We shall consider global (over the whole data set) and local estimates of GCV( $\hat{f})$ to aid 
parameter selection. The global GCV can be estimated after performing $\mathrm{n}$ local regressions at each data point $\mathbf{x}_{\mathrm{i}}$-as:

$$
\operatorname{GGCV}(\hat{f})=\left(\sum_{i=1}^{n} e_{i}^{2} / n\right) /\left(1-\sum_{i=1}^{n} h_{i i} / n\right)^{2}
$$

where $h_{\mathrm{ii}}=\mathbf{z}_{\mathrm{i}}^{\mathrm{T}}\left(\mathbf{Z}_{\mathrm{i}}^{\mathrm{T}} \mathbf{W}_{\mathrm{i}} \mathbf{Z}_{\mathrm{i}}\right)^{-1} \mathbf{z}_{\mathrm{i}} \mathrm{w}_{\mathrm{ii}, \mathrm{i}}, \mathbf{z}_{\mathrm{i}}$ is the augmented $\mathrm{d}^{\prime}$-vector corresponding to $\mathbf{x}_{\mathrm{i}}, \mathbf{Z}_{\mathrm{i}}$ is the augmented matrix of the $k$ nearest neighbors of $\mathbf{x}_{\mathbf{i}}, \mathbf{W}_{\mathbf{i}}$ is the weight matrix for estimation at $\mathbf{z}_{i}$, and $w_{i i, i}$ is the weight applied to $z_{i}$, and where $e_{i}=y_{i}-\hat{f}\left(x_{i}\right)$.

One can select appropriate values of $\mathrm{k}$ and $\mathrm{p}$, as the minimizers of the GGCV value computed in equation 12 for each combination of $\mathrm{k}$ and $\mathrm{p}$. These would be the values of $\mathrm{k}$ and $\mathrm{p}$ that would do well on the average. However, in certain situations (e.g., where the curvature of the target function varies over the data, and where the variance of the noise varies over the range of the data), one may wish to make such choices locally at the point of estimate.

Lall et al (1995) introduced the use of a local GCV score that uses data directly from the local regression at the point of estimate. In this case the errors $e_{i, 1}$ are the residues of the model fitted over the $k$ nearest neighbors of the point $\mathbf{x}_{1}^{*}$, and $\mathbf{W}_{i}$ is the corresponding weight matrix. The trace of the matrix $\mathbf{H}$ in this case is simply d', the number of coefficients fitted. The local GCV score is then given as:

$$
\operatorname{LGCV}_{1}(\hat{f})=e_{1}^{\mathrm{T}} \mathbf{W}_{1} \mathbf{e}_{1} /\left(\left(k-d^{\prime}\right) / k\right)^{2}
$$

The appropriate values of $\mathrm{k}$ and $\mathrm{p}$ can then be obtained as the ones that minimize the local GCV score for the local regression. The $\mathrm{LGCV}_{1}$ value also provides insight into the local predictive error variance. This approach to parameter selection is particularly useful when making a few estimates from a large data set. This can be the case when local regression is used for forecasting a nonlinear time series model, as is done later in this paper.

A problem with the two selectors introduced thus far is that they presume a regular or well behaved sampling of the data $\mathbf{x}_{\mathrm{i}}$, and do not address the issue raised earlier of local data clustering and its effects on the regression. Since, both the global and the local GCV statistic are based on estimation at observed points (which may not coincide very well on average with the points at which we need estimates), they provide only limited insight into the best parameters to use locally, particularly in areas that are sparsely sampled. A modification of the local GCV score that attempts to address this problem is now introduced. 
Comparing equations (7) and (13) we see that they differ by a factor $\left(\mathrm{k}-\mathrm{d}^{\prime}\right) / \mathrm{k}$. In this context, the predictive error variance is merely a penalized version of the error variance for fitting, with a penalty that comes directly from the average weight ( $\left.\mathrm{d}^{\prime} / \mathrm{k}\right)$ ascribed to each data point during the fitting process. Now, following Stuart and Ord (1991, p. 1080), the estimation error variance for an estimate $\hat{y}_{1}$ at a data point $\mathbf{z}_{1}$ is obtained in terms of the "leverage" $h_{1}$ exerted by the point of estimate on the observational data set as:

$$
\operatorname{var}\left(\hat{\mathrm{y}}_{1}\right)=\mathrm{s}_{\mathrm{e}_{1}}^{2} \mathrm{~h}_{1}
$$

where $h_{1}=\left\{\mathbf{z}_{1}\left(\mathbf{Z}_{1}^{\mathrm{T}} \mathbf{W}_{1} \mathbf{Z}_{1}\right)^{-1} \mathbf{z}_{1}^{\mathrm{T}} / \mathrm{k}\right\}$.

The leverage $h_{1}$ for a point of estimate located at the mean of the $k$ nearest neighborhood is $\mathrm{d}^{\prime} / \mathrm{k}$. As one moves away from the center, the leverage value increases. The reader may recall that the confidence limits for linear regression expand as one moves away from the center. This corresponds to the increasing pointwise error variance due to increasing leverage, as in eqn. (14).

We can then define a corresponding measure of local predictive mean square error with consideration of the "leverage" the point of estimate exerts on the data set as:

$$
\operatorname{LGCVLEV}\left(\mathbf{z}_{\mathrm{n}}\right)=\operatorname{LGCV}_{1} \mathrm{~h}_{1}
$$

The LGCVLEV score now has a penalty to account for the degree of extrapolation at the point of estimate relative to the $\mathrm{k}$ nearest neighborhood. If the data are clustered and the point of estimate lies in the middle of the cluster, $h_{1}$ and hence the penalty are the lowest. On the other hand, if the point of estimate is outside the cluster, and one is extrapolating, $h_{1}$ and the penalty will be higher. In this case, if LGCVLEV is used to choose the local $\mathrm{k}$, the value of $\mathrm{k}$ should increase until one or more points outside the cluster enter the local data set. Of course, the inclusion of these points could increase the mean square error. A trade off between symmetrization of the neighborhood and centering it about the point of estimate, and between increasing bias resulting from this growth in the neighborhood size is thus recognized. If symmetrization is not possible (e.g., if the point of estimate lies outside the original data set), the leverage penalty will try to shrink the size of the neighborhood, provided that the LGCV score is not significantly increased. The absolute and relative values of leverage increase as the order $\mathrm{p}$ of the polynomial is increased. Thus this statistic will emphasize lower order fits. Recall from (2) that the approximation error of the scheme depends on both the order of the polynomial and on the size of the neighborhood. 
A simple univariate regression example that shows the performance of LGCV and LGCVLEV for selecting the number of neighbors and polynomial order to use is presented through Figure 2. We see that when the point of estimate lies in a local cluster of data, both measures give the same optimal choice. When the point of estimate, lies in between clusters, LGCV will pick a value of $\mathrm{k}$ that leads to estimates from one of the clusters only, whereas LGCVLEV picks a value that forms the $\mathrm{k}$ nearest neighborhood using the 2 adjoining clusters, in a proportion that depends on the relative distance of $x_{1}$ from the 2 clusters. The behavior of LGCV and LGCVLEV near the boundary of the data set is also different as was indicated earlier. For this example the global GCV choices are the same as those for the local GCV.

Local parameter selection can lead to increased variability in the overall estimation scheme. In practice, we recommend that a number of local estimates at the np desired locations be made. Now one can form the average LGCV or LGCVLEV score as a function of $\mathrm{k}$ and $\mathrm{p}$ across this set of $n$ p prediction points. Determine the $\mathrm{k}$ and $\mathrm{p}$ values that minimize these average scores. If these values are not significantly different from the values obtained locally, it is expedient to use the same $\mathrm{k}$ and $\mathrm{p}$ across all prediction points. Variants of this idea across subregions of data are also useful.

Approximate confidence intervals assuming normally distributed local errors with local error variance $s_{e_{1}}^{2}$ can be obtained for each estimate $\hat{f}\left(\mathbf{x}_{1}\right)$ following Stuart and Ord (1991, p. 1043) as :

$\hat{\mathrm{f}}\left(\mathbf{x}_{1}\right)_{\alpha}=\hat{\mathrm{f}}\left(\mathbf{x}_{1}\right) \pm \mathrm{t}_{\mathrm{x}-\mathrm{d}, 1-\alpha / 2} * \mathrm{~s}_{\mathrm{e}_{1}}$

where $t_{k-d^{\prime}, 1-\alpha / 2}$ is a Student's $t$ variate with $\left(k-d^{\prime}\right)$ degrees of freedom, and significance level $\alpha$.

Note that the above confidence interval is likely to be narrower than it should be, because it does not recognize the variability one should associate with the choice of $k$ and $p$. Some consideration of these factors is possible through the use of LGCVLEV ${ }^{0.5}$ in place of $s_{\varepsilon_{1}}$ in equation 16, since LGCVLEV corresponds to an estimate of error variance that recognizes the price to be paid for estimating the smoothing parameters. Prediction intervals are given as:

$\left(1+h_{1}\right) s_{e_{1}}$ in place of $s_{e_{1}}$, or

$(\mathrm{LGCV}+\mathrm{LGCVLEV})^{0.5}$

A better, but computationally more intensive approach to prediction intervals is provided by Yao and Tong (1994) who directly solve for the $\alpha$ conditional percentiles of $y$ given $\mathbf{x}_{1}$, by considering 
an appropriate asymmetric quadratic loss function that depends on the percentile of interest $\alpha$. 


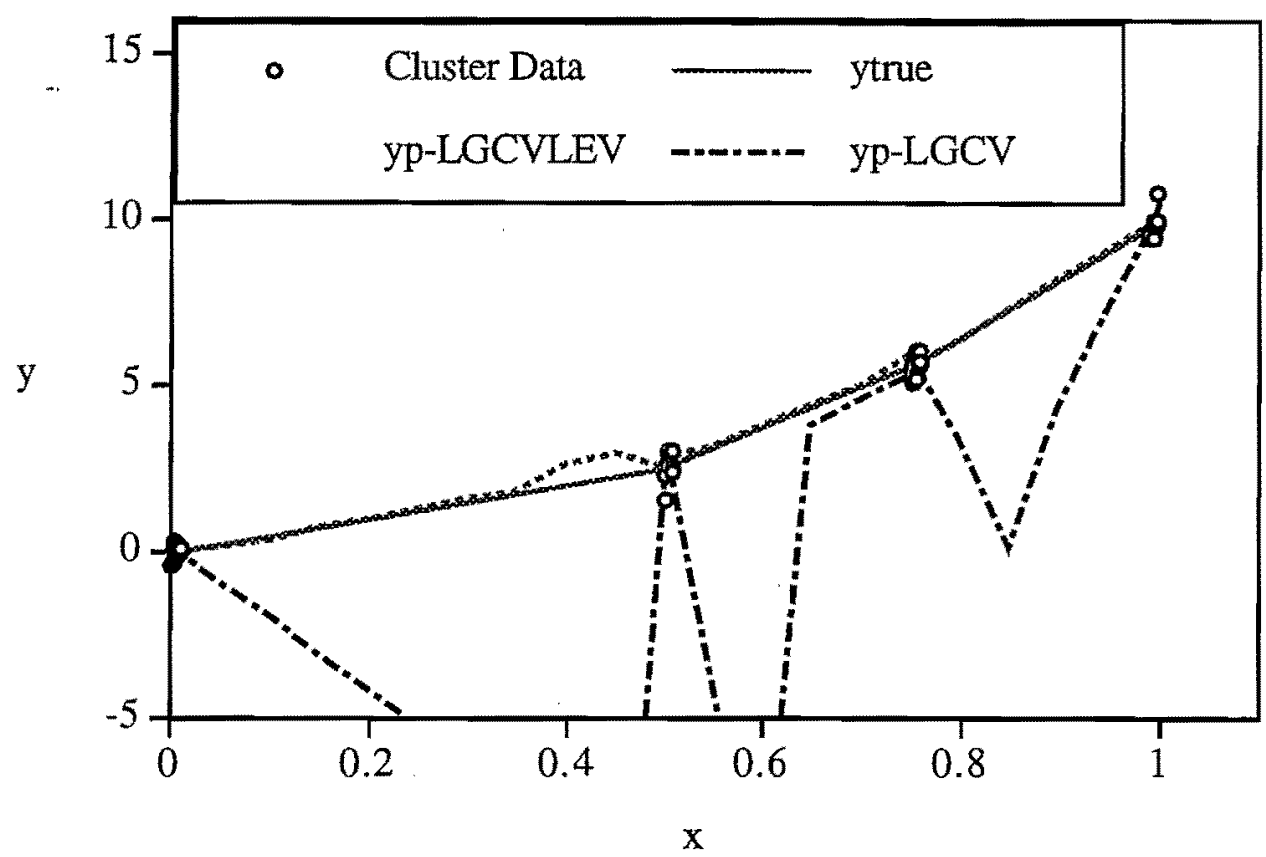

Figure 2

A simple univariate regression example to illustrate how the selection of the number of nearest neighbors, $\mathrm{k}$, varies with LGCVLEV and LGCV for cluster data. A data set of length 40 is generated from the model $y=10 x^{2}+N(0,0.1)$. The sampling locations are organized into four clusters centered at $\left(x_{c}=0.005,0.505,0.755,0.995\right)$, with each cluster spread between $x_{c} \pm 0.005$. Twentyfive points ( 4 at the cluster centers, and 21 equally spaced from 0 and 1) were considered for estimation. Locally linear fits were considered with $\mathrm{k}$ ranging from 4 to 40 . LGCV almost always (23 out of 25 cases) picks $k=5$. GGCV also picks $k=5$, and hence gives the same estimates as LGCV. Since each cluster has 10 points in it, in most cases the resulting estimates will be based only on data from one cluster. The resulting fit is seen to be quite poor except at the estimation points that lie in or very close to the clusters. LGCV estimates that are really poor are off the graph (worst fit is at $\mathrm{x}=0.3$ where the value is -70.2 ). LGCVLEV picks $\mathrm{k}=8$ to 17 for the estimates within clusters, and $\mathrm{k}=17$ to 29 for the estimation points that are not in the clusters. In this case, the data from each cluster is used for estimates within clusters, and data from more than one cluster is used when the point of estimate is in between clusters. The resulting estimates are seen to be quite good over the range of the data. The "bias" in the estimate between $x=0.4$ and $x=0.5$, corresponds to a LGCVLEV choice of $k=29$. Such increased variability is a consequence of the increased number of parameter choices when choosing parameters locally. 


\section{Algorithm}

The Local Polynomial Regression algorithm is now summarized.

I. Given data $\left(\mathbf{x}_{\mathrm{i}}, \mathrm{y}_{\mathrm{i}}\right), \mathrm{i}=1 \ldots \mathrm{n}$.

- Plot Scatterplots of y vs. each component of $\mathbf{x}$. Decide on a range $\{\mathrm{k} 1, \mathrm{k} 2\}$ of nearest neighbors, and $\{\mathrm{p} 1, \mathrm{p} 2\}$ of polynomial order.

- Typically $\mathrm{p} 1=0$ or 1 , and $\mathrm{p} 2$ is 1 or $2 ; \mathrm{k} 1 \geq 2 * \mathrm{~d}$, where $\mathrm{d}$ is the total number of coefficients to solve for, and $\mathrm{k} 2=\mathrm{n}$. The case $\mathrm{p} 1=0$ takes care of locally constant fitting, i.e., kernel regression.

II. For each point of estimate $\mathbf{x}_{1}, 1=1 \ldots \mathrm{np}$, estimate a local regression (eqns 4,5 ) for each value of $\mathrm{k}$ and $\mathrm{p}$ considered.

- Form the augmented $\mathrm{k}^{*} \mathrm{~d}$ matrix $\mathbf{Z}_{1}$ for the polynomial basis indicated by $\mathrm{p}$ with $\mathrm{k}$ nearest neighbors.

- Form the $\mathrm{k}^{*} \mathrm{k}$ matrix of weights $\mathrm{W}_{1}$ indicated by the number of neighbors $\mathrm{k}$.

- Identify for each such regression, the coefficients $\beta_{j}, j=1$..d', and associated columns of the matrix $\mathbf{Z}_{1}$ to retain using (4). Re-solve the model with the the reduced set and compare the LGCV value for the reduced model with the original model. OPTIONALLY adjust the model using LGCV.

- Select optimal $\left(\mathrm{k}^{*}, \mathrm{p}^{*}\right)_{1}$ as the minimizers of LGCVLEV (or LGCV) over $\mathrm{k}$ and $\mathrm{p}$.

- Retain for each such regression, the following statistics:

1) the estimate $\hat{f}\left(\mathbf{x}_{1}\right)$, (2) the residual $e_{1}(k, p)$, (3) $\operatorname{LGCV}_{1}(k, p)$, (4) $\operatorname{LGCVLEV}_{1}(k, p),(5) s_{e_{1}}^{2},(6)$ $h_{i i}$ (eqn 12), and $h_{1}$ (eqn 14), (7) the number of coefficients, $d^{\prime}$ that were selected, and $(8)\left(k^{*}, p^{*}\right)_{1}$ - Provide prediction intervals for $\hat{\mathrm{f}}\left(\mathrm{x}_{1}\right)$ corresponding to $\mathrm{d}^{\prime}, \mathrm{k}^{*}, \mathrm{p}^{*}$ if needed.

III. (OPTIONAL) Compute the following statistics from the information retained in II.:

- $\operatorname{GGCV}(k, p)$ (eqn 12), Average(LGCVLEV ${ }_{1}(k, p)$, Average $\left(\operatorname{LGCV}_{1}(k, p)\right)$. The averages are taken over $1=1$..np.

- Determine the $\mathrm{k}^{*}, \mathrm{p}^{*}$ that minimize (1) GGCV, (2) Average (LGCVLEV) and (3) Average (LGCV). If these are all similar and the spread of $\left(\mathrm{k}^{*}, \mathrm{p}^{*}\right)_{1}$ is relatively small, use these values at all 1 , else use the $\left(\mathrm{k}^{*}, \mathrm{p}^{*}\right)_{1}$ determined in II. 


\section{Application}

The primary application we consider in this paper is the forecast of the volume of the Great Salt Lake at key points in time from its 1847-1993, biweekly time series. However, we shall begin by forecasts of data from two known models to assure ourselves that the forecasting scheme can work. The generic forecasting model is described first.

Let us say that we have a time series, $G_{t}, t=1 \ldots n t$. We shall presume that $G_{t}$ is the outcome of a Markovian (i.e., future values depend only on a finite set of past values) process, and consider a nonlinear, autoregressive model as appropriate for forecasts of such a system. We refer the reader to Tong(1990) for theoretical background and attributes of such an approach. The model of interest is stated as:

$\mathrm{G}_{\mathrm{t}+\mathrm{m}}=\mathrm{f}_{\mathrm{m}}\left(\mathrm{V}_{\mathrm{t}}\right)+\mathrm{e}_{\mathrm{t}}$

where $G_{t+m}$ is an $m$ step ahead forecast, $f_{m}\left(V_{t}\right)$ is a forecast or recursion function (the conditional expectation of $G_{t+m}$ given $V_{t}$ ), that is presumed to be continuous and twice differentiable, $e_{t}$ is a independent and locally (in the $\mathrm{k}$ nearest neighborhood of $\mathrm{V}(\mathrm{t})$ ) identically distributed noise process with zero mean and finite variance, $V(t)$ is a state vector of length $m 1$, with components $\left(G_{t}, G_{t-\tau}, \ldots . G_{t-(m-1) \tau}\right)$, where $\tau$ is a delay or lag between coordinates.

Now, one can consider two approaches for forecasting the $m$ step ahead value for the time series. One could forecast 1 step ahead $m$ times, updating $V_{t}$ to $V_{t+1}, \ldots V_{t+j}$ etc., with the the $j$ forecasted values $G_{t+j}$ that are available at that point. This is called an iterated forecast. Alternately, one could directly forecast $m$ steps ahead using the current $V_{t}$. In either case, $m$ successive estimates of $f_{m}\left(V_{t}\right)$ are needed. We have explored both strategies in our work, and found them to give comparable results for the data sets tested. In what follows, the direct prediction method is described. Iterated forecasts follow a very similar strategy.

Direct Forecasts of a Time Series for $m$ steps forward from time index $t=n t$ :

(1) Select a lag $\tau$ and an embedding dimension $\mathrm{m} 1$. These are parameters that can be varied and picked as the ones that minimize the GGCV for the $m$ step forecasts $G_{t+m}$, or they can be chosen based on other prescriptive criteria (e.g., $\tau$ as the delay that corresponds to the first minimum of the Average Mutual Information, and $\mathrm{m} 1$ as the value that minimizes the percentage of false nearest neighbors - see Abarbanel et al (1993) for details of both prescriptions). Usually, these will not be 
changed during the $\mathrm{m}$ step prediction, but may change if the forecasts are started under rather different conditions.

(2) Form a data matrix $\mathbf{X}$ with $\mathrm{m} 1$ columns corresponding to $\mathrm{V}_{\mathrm{t}}$, and (nt-(m1-1)* $\tau-\mathrm{m}$ ) rows from the time series $G_{t}$ using the appropriate lags of the time series. For example say $\tau=2, m l=3$. Then the first row of $\mathbf{X}$ will have $\mathrm{G}_{5}, \mathrm{G}_{3}, \mathrm{G}_{1}$; and the last row will have $\mathrm{G}_{\mathrm{nt}-\mathrm{m}}, \mathrm{G}_{\mathrm{nt}-\mathrm{m}-2}, \mathrm{G}_{\mathrm{nt}}$ $\mathrm{m}-4$.

(3) Form a data vector $\mathbf{y}$ corresponding to $\mathbf{X}$, as the value $G_{t+m}$ corresponding to each row of the first column of $\mathbf{X}$. For example, for $m=5$ in the previous example, the first entry of $y$ is $G_{10}$, and the last entry is $\mathrm{G}_{\mathrm{nt}}$.

(4) Solve the local regression problem at $x_{1}=\left(G_{n t}, G_{n t-\tau}, \ldots G_{n t-(m-1) \tau}\right)$ as in Section 4.

(5) Evaluate the forecast and provide confidence/prediction intervals if needed.

Note that since $\mathrm{V}_{\mathrm{t}}$ does not change for each step in the direct forecasting method, if $\mathrm{k}$ and $\mathrm{p}$ do not change one can retain the matrix $\left(\mathbf{Z}_{1}^{\mathrm{T}} \mathbf{W}_{1} \mathbf{Z}_{1}\right)^{-1}$ between forecasts, and speed up the computations.

Synthetic Series:

The first scenario is a time series of length 200 from an $A R(2)$, or autoregressive model of lag 2. The model is defined by:

$\mathrm{y}_{\mathrm{t}}=\mathrm{y}_{\mathrm{t}-1}-0.5 \mathrm{y}_{\mathrm{t}-2}+\varepsilon_{\mathrm{t}}$

where $\varepsilon_{\mathrm{t}}$ is a Normally distributed random variable with mean 0 and variance 1 .

In this case, we selected $\tau=1$, and varied $\mathrm{m} 1$ from 1 to 5 . The number of nearest neighbors to use was varied from 50 to the sample length 200 , and linear and quadratic (without cross product terms) fits were considered from 1 to 20 various points in the series. In all cases LGCVLEV was used to select the parameters of interest. The number of nearest neighbors was consistently picked as the full sample size, $\mathrm{ml}$ was typically picked to be 2 , and linear fits were always selected. Since, the models selected were essentially global, linear, autoregressive models each time, the resulting statistical properties were satisfactory.

\section{Lorenz Equations:}

The Lorenz equations are given as: 


$$
\begin{aligned}
& \dot{x}=-\sigma(x+y) \\
& \dot{y}=-x z+r x-y \\
& \dot{z}=x y-b z
\end{aligned}
$$

Here we took $\sigma=16, \mathrm{r}=45.92, \mathrm{~b}=4$, and $\partial \mathrm{t}=0.05$, and sampled the $\mathrm{x}$ state variable. This is a chaotic system, that has been well studied by many investigators. From prior work (Lall et al, 1995a, Moon et al, 1995) we knew that one should expect $\tau=2$ to 4 , and $\mathrm{m} 1=4$ to 6 . Consequently we investigated these values and $\mathrm{k} 1=50$ to $\mathrm{k} 2=150$, and $\mathrm{p} 1=1, \mathrm{p} 2=2$. Forecasts from index 1996 of the $\mathrm{x}$ time series are presented in Figure 3. No data after index 1996 were used for the forecasts.

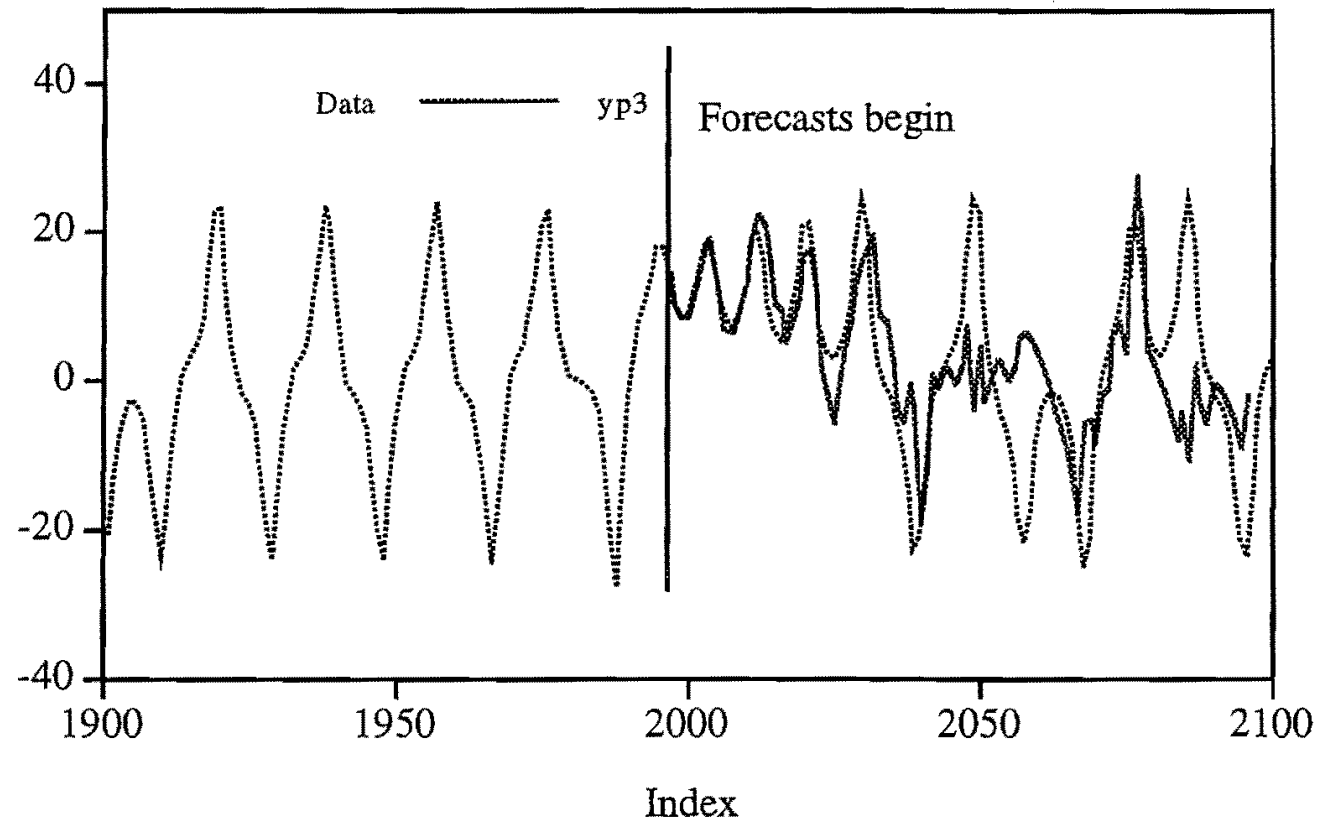

Figure 3

Direct m-step forecasts of Lorenz data starting from index 1997. The dotted line represents the actual values while the solid lines are the forecasted values $(m 1=5, \tau=3, k=50$ at the first 21 points, 90 or 150 at the rest of the points, and $\mathrm{p}=1$ at the first 9 points, $\mathrm{p}=2$ at the rest points). The divergence of the forecasted and the observed trajectories near index 2030, is characteristic of the loss of predictability in the Lorenz system as trajectories pass near the unstable point $(x=y=z=0)$. The increase in $k$ and $p$ after the first 20 points may reflect increasing derivatives of $f\left(V_{t}\right)$ as one approaches the origin. 
The Lorenz system has an instability near $\mathrm{x}=\mathrm{y}=\mathrm{z}=0$. Trajectories that approach this state tend to diverge rapidly. We notice from Figure 3 that the forecasts of the Lorenz $x$ variable are quite good until the trajectory passes near the unstable point. A small uncertainty in the value at index 1996 leads to the trajectories from the numerical simulation of the Lorenz equations diverge similarly. Thus this divergence is intrinsic to this model. Subsequent similarity in the forecast and actual trajectories is coincidental. Similar results were obtained in Lall et al (1995) using MARS. The local polynomial forecasts are however considerably faster to execute.

Great Salt Lake Forecasts:

The Great Salt Lake (GSL) of Utah is a closed lake in the lowest part (elevation $1280 \mathrm{~m}$. above Mean Sea Level) of the Great Basin (latitudes $40^{\circ} 20^{\prime}$ and $41^{\circ} 40^{\prime} \mathrm{N}$, and longitudes $111^{\circ}$ $52^{\prime}$ and $113^{\circ} 06^{\prime} \mathrm{W}$ ), in the arid Western U.S.A. The GSL is approximately $113 \mathrm{~km}$ long and $48 \mathrm{~km}$ wide, with a maximum depth of $13.1 \mathrm{~m}$ and an average depth of $5.0 \mathrm{~m}$. The large surface area and shallow depth make the lake very sensitive to fluctuations in long term climatic variability. As shown in Figure 4, the lake volume has varied considerably over decadal time scales during the last 140 years. The low frequency character makes this an interesting time series to forecast. 


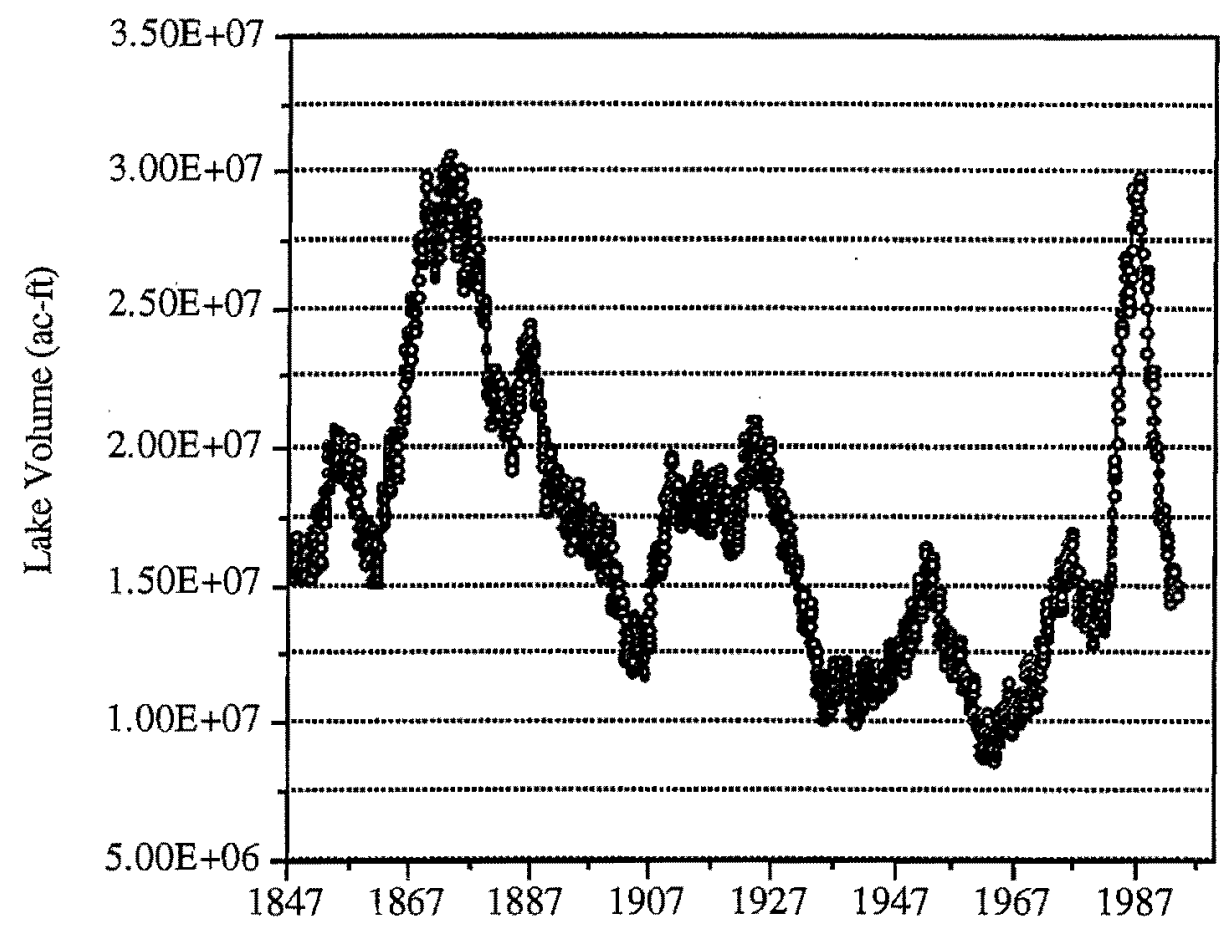

Figure 4

Biweekly time series of the Great Salt Lake, 1847-1993

We considered blind forecasts of the GSL volume from different states for 1 to 2 years into the future from the date of forecast. The forecasted values are then compared with the volumes that were actually recorded subsequently. They are presented in Figures 5 and 6 . The lag $\tau$ was selected as 10 as in the range of the first minimum of the average mutual information (Moon et al, 1995) and it was based on experimentation to get the best predictions (min predictive squared error). An embedding of $\mathrm{ml}=5$ was selected after experimentation with various values in the range 1 to 9 . Usually, this value corresponded to the one that minimized GGCV, LGCV or LGCVLEV. 


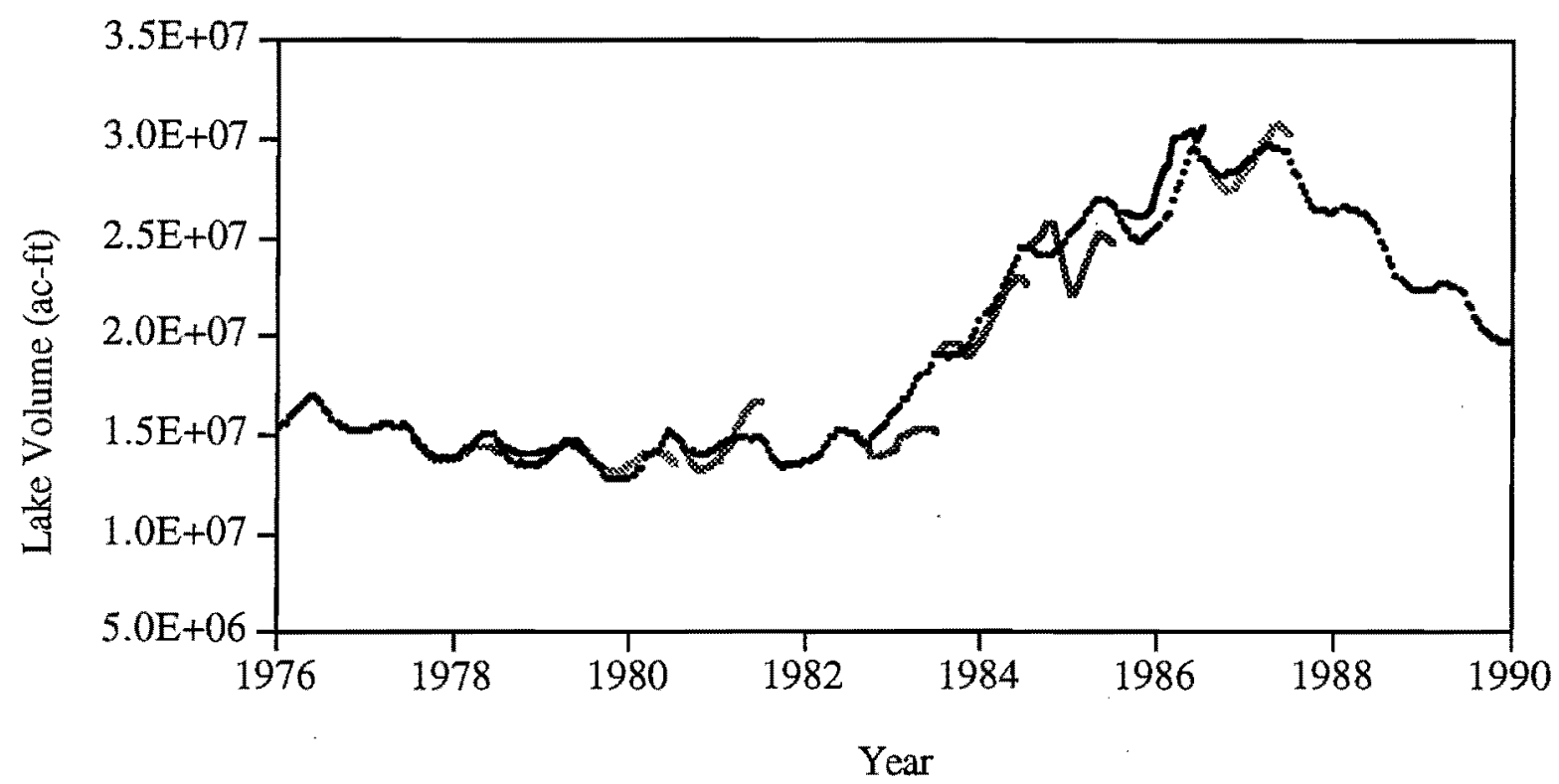

Figure 5

A sequence of 1 year blind forecasts of the GSL using the direct m step method, from August 1977 to July 1987. The dots represent the observed GSL time series. The solid lines represent 12 forecasts, one for each month of the next year. Only data available up to the beginning of the forecast period is used for fitting and forecasting. Given the extreme nature of the 1983-87 period the predictions appear to be quite good. Of particular interest is the forecast starting in August 1983. The predictability is quite poor for this forecast. 


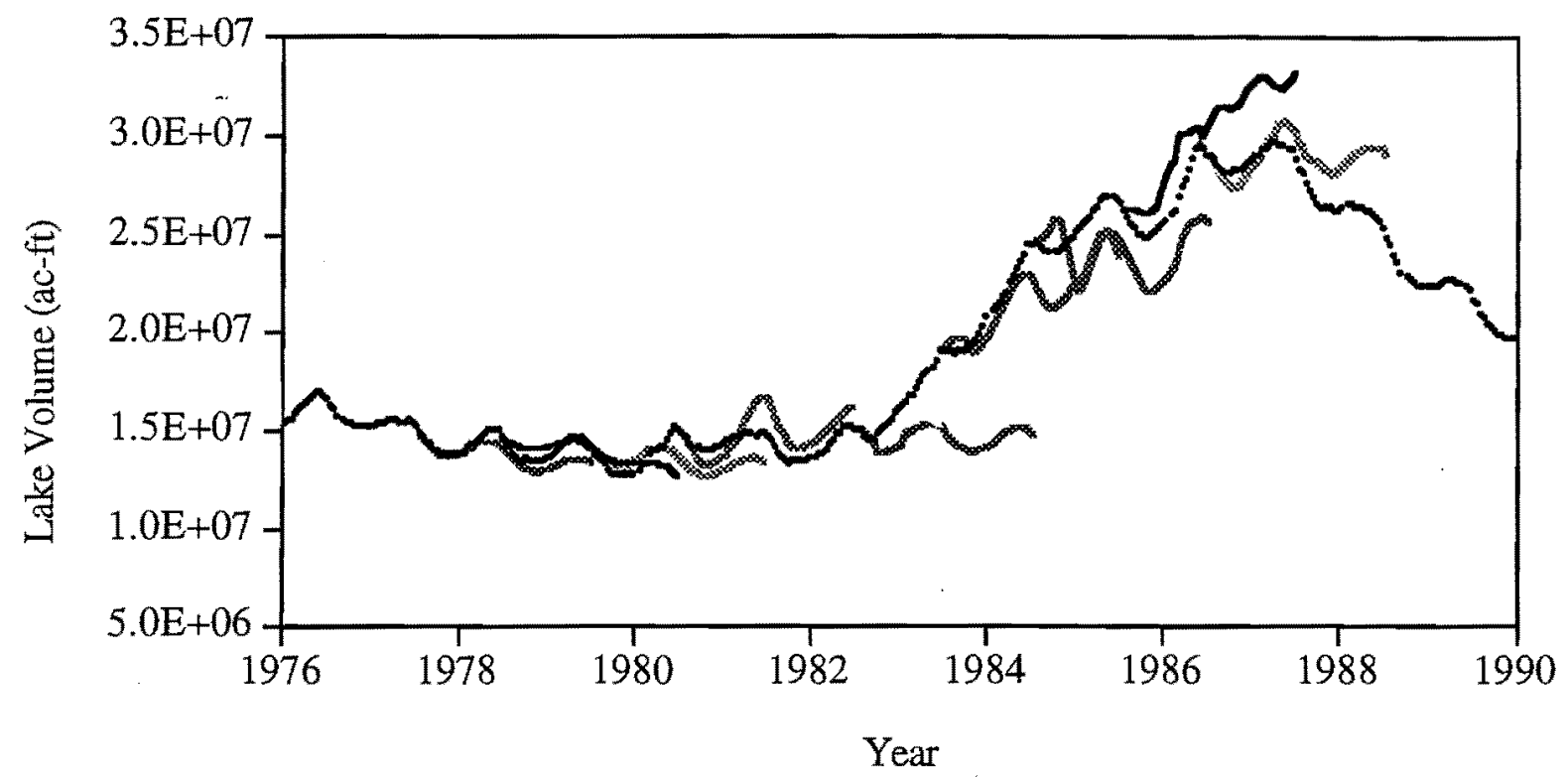

Figure 6

A sequence of 2 year blind forecasts of the GSL using the direct m step method, from August 1977 to July 1988. The dots represent the observed GSL time series. The solid lines represent 24 forecasts, one for each month of the next year. Only data available up to the beginning of the forecast period is used for fitting and forecasting. Comparing with Figure 5, we see that some trajectories (e.g., August 1980 start, August 1983 start) that appeared to be departing from the observed trajectory at the end of 1 year have followed the observed trajectory during the next year.However, others (e.g., August 1982 start) have continued to drift away. The forecast started in August 1981 does quite well till it reaches August 1982 when it behaves much like the first year of the forecast started in August 1982. The second year of the forecast started in August 1985 diverges from the observed trajectory, while the forecast with information up to August 1986 does much better. Clearly, predictability is quite high for the conditions in the 1970's through 1982. In 1982 , the system appears to pass through a rather unpredictable state. The predictability of the system during the extreme event seems to be not as good as during the 1970's, but still quite good. 
We searched over $k 1=50$ to $k 2=150$ nearest neighbors and typically selected 120 to 150 . Locally linear and quadratic (without $\mathrm{x}$-products) fits were considered. Typically linear was selected. The results are discussed in the figures.

Finally, a forecast of the Great Salt Lake volume for 4 years from the end of the available record is presented in Figure 7. Prediction intervals for this forecast were calculated using equation (17).

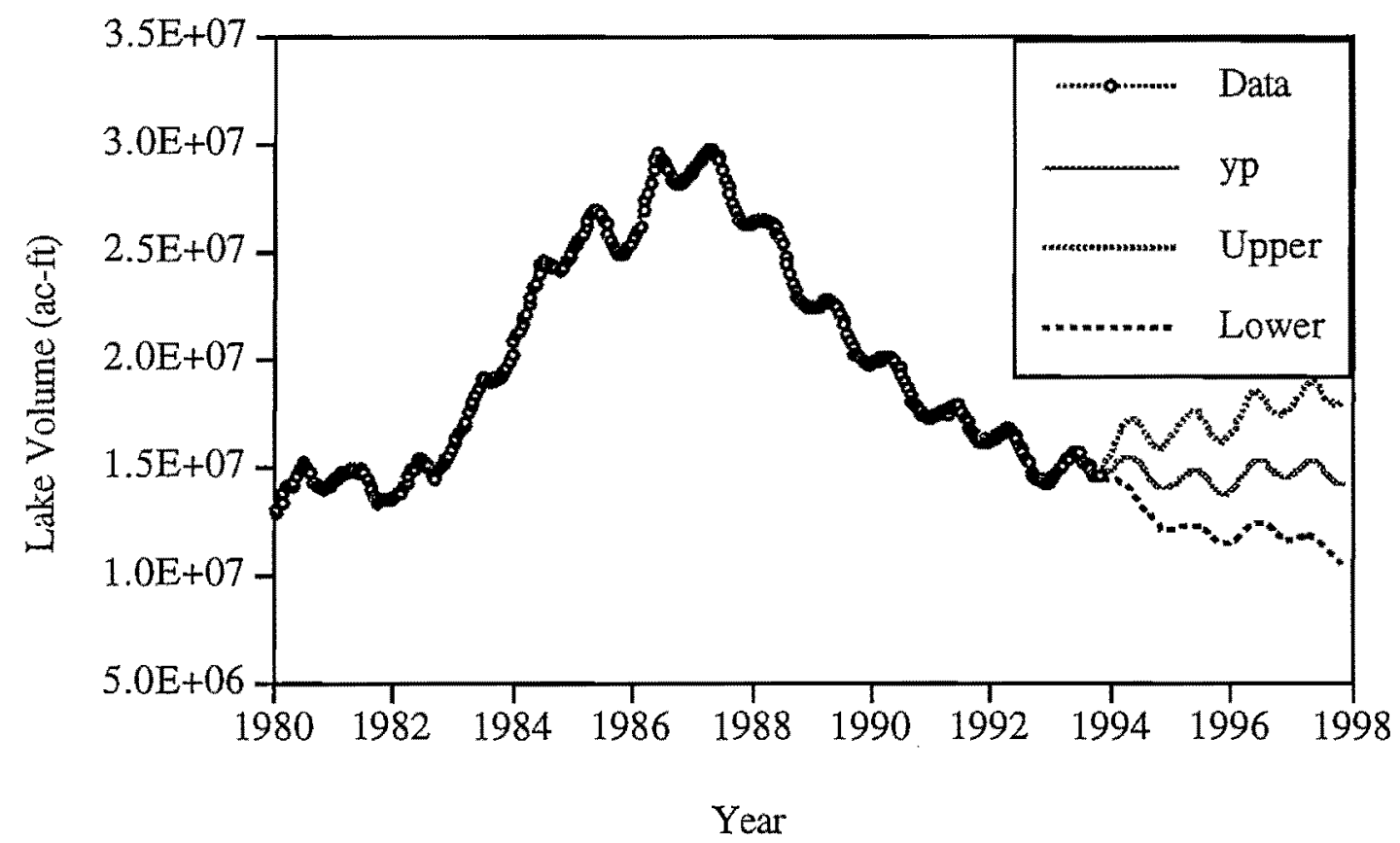

Figure 7

Forecasts for 4 years starting Aug. 1993. The solid line is the forecasted sequence while circles are the actual series. Prediction intervals using LGCVLEV for the forecast are also shown.

\section{Conclusions}


A locally weighted polynomial regression methodology for approximating nonlinear regressions was introduced in this paper. The basic algorithm presented is derived from the work by Cleveland and Devlin (1988), and Lall et al (1995). A new, local cross validatory criteria was introduced for selecting the smoothing parameters of the method while considering a bias-variance trade off in estimation and symmetrization of the $\mathrm{k}$ nearest neighborhood of the point of estimate. The utility of this selector for this purpose was demonstrated with a simple example.

From Taylor series, the approximated error of a local linear model with small $\mathrm{k}$ is comparable to a local quadratic model with large $\mathrm{k}$. So, if $\mathrm{n}$ is much greater than $\mathrm{d}$ and $\mathrm{f}$ " is high then a local quadratic model will be picked. If $\mathrm{n}$ is not much greater than $\mathrm{d}$ then a local linear model is picked.

The methodology presented was then applied to the forecast of selected time series with encouraging results. These methods are still evolving. We can expect improvements in procedures for estimating prediction intervals and for selecting parameters of the method. Algorithmic improvements for more efficiently exploiting multivariate data structures are also to be expected.

From a hydrological point of view, these methods provide new directions for the exploration of data as well as the possibility of dramatic improvements in time series forecasting and spatial surface reconstruction. The latter is discussed at length in Lall et al (1995b), and compared with Kriging.

\section{Acknowledgements}

Partial support of this work by the USGS grant \# 1434-92-G-226 and NSF grant \# EAR-9205727 is acknowledged.

\section{References}

Abarbanel, H.D.I., R. Brown, J.J. Sidorowich, and L.S. Tsimring, The Analysis Of Observed Chaotic Data In Physical Systems, Rev. of Modern Phys., 65 (N4), 1331-1392, 1993.

Abarbanel, H.D.I.and U. Lall, Nonlinear Dynamics of the Great Salt Lake: System Identification and Prediction, Clim. Dyn., (submitted), 1995. 
Cleveland, W.S., Robust locally weighted regression and smoothing scatterplots, J. Amer. Stat. Assoc., 74 (368), 829-836, 1979.

Cleveland, W.S., S. J. Devlin, Locally Weighted Regression: An Approach to Regression Analysis by Local Fitting, J. Amer. Stat. Assn., 83 (403), 596-610, 1988.

Cleveland, W.S., S. J. Devlin, E. Grosse, Regression by Local Fitting, J. Econometrics, 37, 87-114, 1988.

Craven, P. and Wahba, G., Smoothing noise data with spline functions, Numerische Mathematik, 31, 377-403, 1979.

Efron B. and R.J. Tibshirani, An introduction to the Bootstrap, Chapman and Hall, 1993.

Eubank, R., Spline smoothing and nonparametric regression, Marcel Dekker, New York, 1988.

Fan, J. and I. Gijbels, Variable bandwidth and local linear regression smoothers, Ann. Statist. 20, pp196-216, 1993.

Friedman, J.H., Multivariate adaptive regression splines, , 19 (1), 1-141, 1991.

Härdle, W. and T.M. Stoker, Investigating smooth multiple regression by the method of average derivatives, J. Amer. Statist. Assoc. 84, pp986-95., 1989.

Härdle, W., Applied Nonparametric Regression, in Econometric Society Monographs, pp. 333, Cambridge University Press, Cambridge, 1989.

Härdle, W., How to determine the bandwidth of some nonlinear smoothers in practice, Lecture Notes in Statistics 26, pp163-184, Springer-Verlag, 1984.

Hastie, T.J. and C. Loaser, Local regression: automatic kernel carpentry (with discussion), Statist. Sci. 8, pp120-43, 1993.

Helsel, D.R. and R.M. Hirsch, Statistical methods in water resources, Elsevier, New York, 1992.

Lall, U., Nonparametric function estimation: Recent hydrologic contributions, Contributions in hydrology, U.S. National report to the IUGG 1991-1994, 1994.

Lall, U., T. Sangoyomi, H.D.I. Abarbanel, Nonlinear dynamics of the Great Salt Lake: Nonparametric short term forecasting, Water Resources Research, (to appear), 1995.

Lall, U., K. Bosworth, and A. Owosina, Local Polynomial Estimation of Spatial Surfaces, Comp. Stat., (to appear), 1995a. 
Lall, U., T. Sangoyomi, and H.D.I. Abarbanel, Nonlinear Dynamics Of The Great Salt Lake: Nonparametric Short Term Forecasting, Water Res. Res., (to appear), 1995 b.

Lejeune, M., Estimation non-parametrique par noyaux: regression polynomial mobile, Rev. Stat. App1. 33, pp43-67, 1985.

Li, K.-C., From stein's unbiased risk estimates to the method of generalized cross validation, The annals of statistics $13(4), \mathrm{pp} 1352-1377,1985$.

Macauley, F.R., The smoothing of time series, National Bureau of Economic Research, New York, 1931.

Moon, Y.-I., B. Rajagopalan, U. Lall. (1995). "Estimation of Mutual Information Using Kernel Density Estimators." Physical Review Letters, to appear.

Müller, H.-G., Weighted local regression and kernel methods for nonparametric curve fitting, J. Amer. Statist. Assoc. 82, pp231-238, 1987.

Press W. (1989). Numerical recipes : the art of scientific computing. Cambridge University Press.

Sangoyomi, T.B., Climatic Variability and Dynamics of Great Salt Lake Hydrology, PhD. dissertation , 247 pgs. thesis, Utah State University, Logan, Utah., 1993.

Scott, D.W., Multivariate Density Estimation, John Wiley and Sons, New York, 1992.

Silverman, B.W., Density estimation for statistics and data analysis, Chapman and Hall, London, 1986.

Stuart, A. and J. K. Ord, Kendall's Advanced Theory of Statistics. New York, Oxford University Press, 1991.

Tong, H., Non-linear time series: A dynamical system approach, Oxford Science Publications, 1990 .

Wand, M. P. and M. C. Jones, Kernel smoothing, Chapman and Hall, 1995.

Yao, Q. and H. Tong, On prediction and chaos in stochastic systems, Phil. Trans. R. Soc. Lond. A 348 , pp. 357-369, 1994. 